\title{
CT facilitates improved diagnosis of adult intestinal malrotation: a 7-year retrospective study based on 332 cases
}

\author{
Ziman Xiong ${ }^{1}$, Yaqi Shen ${ }^{1 *} \mathbb{C}$, John N. Morelli ${ }^{2}$, Zhen $\mathrm{Li}^{1}$, Xuemei Hu${ }^{1}$ and Daoyu Hu${ }^{1}$
}

\begin{abstract}
Objective: To classify adult intestinal malrotation by $\mathrm{CT}$.

Methods: This retrospective study enrolled adults diagnosed with intestinal malrotation who underwent abdominal CT at our institution between June 1, 2013, and August 30, 2020. All patients' clinical information was recorded. Patients were divided into groups undergoing surgical and conservative management. The duodenum (nonrotation, partial rotation, and malrotation), jejunum, cecum, and the superior mesenteric artery/superior mesenteric vein relationship were reviewed on the $\mathrm{CT}$ images of each patient, and classification criteria developed based on the first three items. For each patient, each item was assessed separately by three radiologists. Consensus was required from at least two of them.
\end{abstract}

Results: A total of 332 eligible patients (218 men and 114 women; mean age $51.0 \pm 15.3$ years) were ultimately included and classified into ten types of malrotation. Duodenal partial rotation was present in most $(73.2 \%, 243 / 332)$ with only $25 \%$ (83/332) demonstrating nonrotation. The jejunum was located in the right abdomen in $98.2 \%$ (326/332) of cases, and an ectopic cecum was found in only 12\% (40/332, 29 cases with a left cecum, 7 pelvic, and 4 at midline). Asymptomatic patients comprised 56.6\% (188/332) of cases, much higher than that in previous studies (17\%, $n=82, p<.001)$, comprised mainly of patients with duodenal partial rotation $(80.3 \%, 151 / 188)$. In 91 patients with detailed clinical data available (12 managed surgically and 79 conservatively), a significant difference in malrotation CT categorization was identified $(p=.016)$.

Conclusions: $\mathrm{CT}$ enables greater detection of asymptomatic intestinal malrotation, enabling classification into multiple potentially clinically relevant subtypes.

Keywords: Intestinal malrotation, Adults, Computed tomography, Diagnosis

\section{Key points}

- CT allows detection asymptomatic intestinal malrotation.

- Difference was observed in CT classification between the surgical and conservative groups.

\footnotetext{
*Correspondence: yqshen@hust.edu.cn

${ }^{1}$ Department of Radiology, Tongji Hospital, Tongji Medical College, Huazhong University of Science and Technology, 1095 Jiefang Avenue, Qiaokou District, Wuhan 430030, Hubei, China

Full list of author information is available at the end of the article
}

- Classification of intestinal malrotation enables identification of high-risk patients.

\begin{abstract}
Introduction
Intestinal malrotation is a congenital anomaly, often considered a pediatric disorder since most patients are diagnosed within the first year of life [1]. Definitive diagnosis can be made by typical symptoms of bilious vomiting in combination with an abnormal upper gastrointestinal (UGI) series [2-4]. In adult patients, however, the
\end{abstract}


diagnosis is often incidental. Because of atypical symptoms, these patients are often initially diagnosed with other diseases [5] with further imaging studies or surgery revealing intestinal abnormalities. In fact, the number of adult malrotation patients is far underestimated. Nehra et al. [6] found adults accounting for $48 \%$ (82/170) of malrotation cases with $17 \%$ of these asymptomatic. However, being asymptomatic at a given point in time does not guarantee a patient will remain asymptomatic for life. In some cases, coexisting diseases or changes in physical condition could trigger a series of complications, including life-threatening volvulus $[7,8]$. There are a lack of research on asymptomatic patients and still no clear indication which patients may develop dangerous complications in the future requiring surgery $[9,10]$.

The UGI series remains the current gold standard for the diagnosis of intestinal malrotation and is widely used in pediatrics [11]. In addition, ultrasound (US) is also recommended for the screening of malrotation with volvulus [12]. Some studies have been conducted in pediatric patients using UGI or US [13-16]. However, in adult patients, computed tomography (CT) scans, especially with intravenous and oral contrast, have greater diagnostic value, and some studies recommend $\mathrm{CT}$ as the first choice in adult patients with suspected malrotation $[5,8$, 17]. CT not only displays abnormal signs visible on UGI and US, but also avoids the influence of intestinal gas, enabling acquisition of complete anatomical information regarding the bowel. There have also been studies using CT to classify intestinal variation in adult patients [18, 19], but these included fewer than 20 patients. In addition, the literature on adult intestinal malrotation mostly consists of case reports.

In order to improve the awareness of radiologists and clinicians about adult intestinal malrotation, in the current study, records from all adult patients diagnosed with intestinal malrotation at our institution were reviewed since 2013 and analysis of relevant clinical data as well as CT images performed.

\section{Materials and methods Subjects}

This retrospective study was approved by the local institutional review board, and informed consent was waived. The electronic medical record system in our institution between June 1, 2013, and August 30, 2020, was reviewed using "intestinal malrotation" and "midgut malrotation" as keywords for searching. A series of inclusion/exclusion criteria were constructed to select eligible cases followed by further analysis.

Criteria for selecting the subjects were as follows: (a) adult patients (age $\geq 18$ years old); (b) intestinal malrotation confirmed by surgery or by consensus among three radiologists (Additional file 1: Methods); (c) abdominal CT images available; and (d) clinical data available (at least sex, age, and symptoms at initial diagnosis). Exclusion criteria were: (a) CT images unavailable or the scan not including the entire abdomen; (b) CT scan performed after small bowel surgery; (c) clinical data unavailable; and (d) malrotation ruled out after review by a senior radiologist.

Clinical data of patients meeting the above criteria were reviewed (sex, age, symptoms at initial diagnosis, duration of symptoms, comorbidities, and treatment), and the patients were divided into groups based on whether they underwent surgical or conservative management. Further analysis of these two groups was then performed.

\section{Image acquisition}

All patients underwent abdominal $\mathrm{CT}$ at our institution. Vendors of CT equipment included GE Healthcare (Lightspeed 16, Lightspeed 64, Discovery 750, and Brightspeed Elite, Waukesha, WI, USA), Philips Healthcare (Brilliance 16 and ICT256, Amsterdam, the Netherlands), and Toshiba Medical Systems (Aquilion One, Otawara, Japan). The CT tube voltage was 120 or $140 \mathrm{kVp}$, and the automatically controlled tube current was 110-720 mAs. The CT scan covered from the diaphragm to the pubic symphysis. If possible, fast for 4-6 hours before the scan, followed by ingestion of $1000-1500 \mathrm{ml}$ of $2.5 \%$ mannitol water solution orally $40-60 \mathrm{~min}$ prior to the scan. Some patients underwent an unenhanced CT, followed by contrast-enhanced imaging; others were scanned with IV contrast only. Contrast-enhanced CT was performed after a rapid bolus of iopromide (Ultravist $370,370 \mathrm{mg} /$ $\mathrm{mL}$, Bayer Schering Pharma, Berlin, Germany) $(1.5 \mathrm{~mL} /$ $\mathrm{kg}$ ) at a rate of $3-5 \mathrm{~mL} / \mathrm{s}$, followed by a $20-\mathrm{mL}$ saline flush using a power injector. Images were routinely obtained in the arterial, intestinal, or portal venous phases at 30, 45, or $70 \mathrm{~s}$, respectively.

\section{Image interpretation}

CT images of all patients were evaluated separately by three radiologists. For each patient, at least two radiologists had to agree on each item of evaluation. The assessment included:

1. Duodenum crossing the midline (the space between the aorta and the superior mesenteric artery (SMA)) or not (a duodenum failing to cross the midline was considered duodenal nonrotation, a duodenum crossing midline then suddenly returning to the right of midline was considered duodenal partial rotation, and a duodenum crossing midline without folding back was considered duodenal malrotation),

2. The main site of the jejunum, 
3. The location of the cecum,

4. Position of SMA relative to superior mesenteric vein (SMV),

5. The presence of the whirlpool sign.

CT classification of patients was based on (1)-(3) (Fig. 1).

\section{Statistical analysis}

All statistical analysis was performed with SPSS 26.0. To compare results with those of previous studies and to compare variables between the two groups herein, the independent Student t-test was used for continuous variables and Chi-square or Fisher's exact test for categorical variables. All analyses were considered significant at $p$ values of less than 0.05 (two-tailed).

\section{Results}

A final 332 eligible adult patients with intestinal malrotation were included in the study. Since 188 of these patients were incidentally identified and did not exhibit symptoms related to intestinal malrotation or receive relevant treatment, they were included in the asymptomatic group, and the course of their treatment was not further documented. Of the 144 patients whose symptoms were recorded, 8 patients had comorbidities that may be associated with symptoms (gastroptosis, liver cysts, irritable bowel syndrome, ileocecal tumors, rectal cancer). Since the possibility that the symptoms were caused by malrotation could not be ruled out, these symptoms were still recorded. In addition, treatment information was not available for 53 outpatients. Therefore, detailed clinical and treatment information was available for 91 patients, who were categorized into surgical and conservative management groups for further analysis (Additional file 1: Fig. S1).

\section{Clinical characteristics of all cases}

The clinical characteristics of all cases are summarized in Table 1.

An overall male predominance in both groups was observed (male-to-female ratios were: overall 1.91:1, surgical group 2:1, and conservative group 1.47:1) and the age range of included patients was broad (range 18-97). The proportion of asymptomatic patients in the current cohort was $56.6 \%(188 / 332)$, while the proportion of patients with volvulus was $2.1 \%$ (7/332). Abdominal pain was still the main complaint among all patients, and chronic symptoms (complaints lasting over one month) were observed in more than half of patients $(n=91$, $54.9 \%$ ) with some patients experiencing symptoms for years or even decades. The proportion of patients undergoing surgery was $13.2 \%$ (12/91).

\section{CT findings of all cases}

The CT findings of all patients are summarized in Table 2.

CT images of 332 patients were categorized into ten types of malrotation based on the orientation of the duodenum, jejunum, and cecum (Fig. 2). The majority of patients showed duodenal partial rotation $(73.2 \%$, 243/332) (4a-b, 5, 6, and 7 in Fig. 2) and only $25.0 \%$ (83/332) duodenal nonrotation (1a-b, 2, and 3 in Fig. 2).

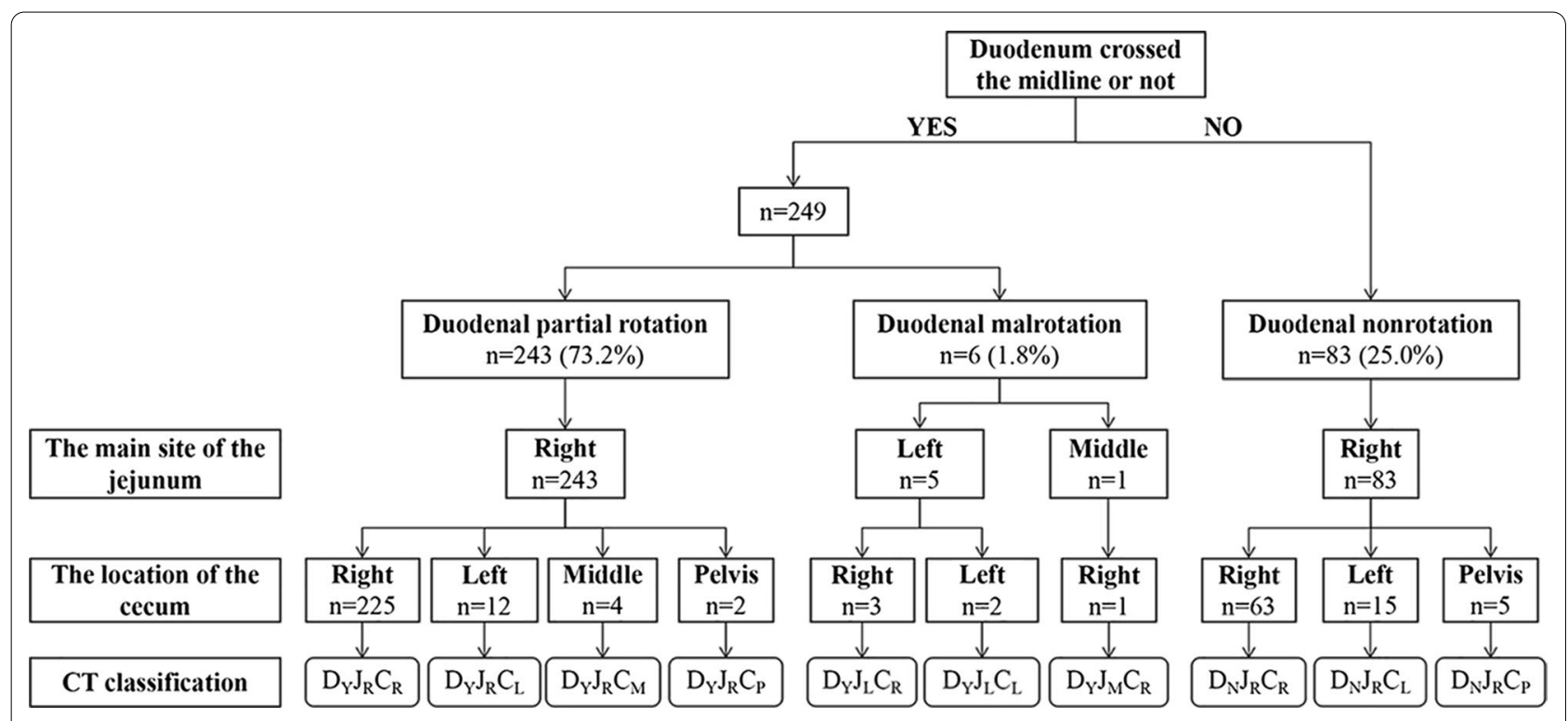

Fig. 1 Process and results of classification of abdominal CT images 
Table 1 Clinical characteristics of 332 adult malrotation patients

\begin{tabular}{|c|c|}
\hline Parameters & $\begin{array}{l}\text { Total }(n=332) \\
\text { No. }(\%)\end{array}$ \\
\hline Gender ratio (male/female) & $1.91: 1$ \\
\hline \multicolumn{2}{|l|}{ Age, years } \\
\hline Mean & $51.0 \pm 15.3$ \\
\hline Range & $18-97$ \\
\hline Asymptomatic patients & $188(56.6)$ \\
\hline \multicolumn{2}{|c|}{ Symptoms at diagnosis ${ }^{\dagger}(n=144)$} \\
\hline Abdominal pain & $114(79.2)$ \\
\hline Bloating & $37(25.7)$ \\
\hline Vomiting & $18(12.5)$ \\
\hline Nausea & $7(4.9)$ \\
\hline Diarrhea & $6(4.2)$ \\
\hline Constipation & $4(2.8)$ \\
\hline Complication: volvulus & $7(2.1)$ \\
\hline \multicolumn{2}{|c|}{ Duration of symptoms ${ }^{\#}(n=91)$} \\
\hline Hours/days & $25(27.5)$ \\
\hline Weeks & $16(17.6)$ \\
\hline Months & $24(26.4)$ \\
\hline Years & $26(28.5)$ \\
\hline \multicolumn{2}{|l|}{ Treatment ${ }^{\#}(n=91)$} \\
\hline Conservative treatment & $79(86.8)$ \\
\hline Surgical treatment & $12(13.2)$ \\
\hline
\end{tabular}

† Patients' first symptoms are duplicated (coexistence of several symptoms)

\# Duration of symptoms and treatment modalities were obtained from 91 patients, and outpatients without detailed records and asymptomatic patients with predominantly other conditions (kidney stones, cirrhosis, hypertension, etc.) were not recorded

In both scenarios, the jejunum was typically located in the right abdomen. For the remaining six patients who exhibited duodenal malrotation, the duodenum crossed the midline and did not fold back $\left[D_{Y} J_{L} C_{R}\right.$ (Fig. 2, type 8), $D_{Y} J_{L} C_{L}$ (Fig. 2, type 9), $D_{Y} J_{M} C_{R}$ (Fig. 2, type 10)]; however, the anatomy in such cases was still abnormal. Overall, most patients exhibited a normal SMA/SMV relationship $(91.6 \%, 304 / 332)$. Among these, SMV angulation followed by a right-sided deviation was most common $(98.7 \%, 300 / 304)$, whereas a straight inferior course was noted in four cases $\left(D_{Y} J_{L} C_{R}, D_{Y} J_{M} C_{R}\right)$. However, SMV angulation was not seen in patients with an abnormal SMA/SMV relationship, all of whom showed SMV coursing directly to the right or left abdomen (Fig. 3). In addition, the "whirlpool sign" was found in 11 patients, including six cases with volvulus, four asymptomatic cases, and one outpatient (Fig. 4).

\section{Comparison of the surgical and conservative groups}

The clinical and CT characteristics of surgically and conservatively managed groups are shown in Table 3 .
Table 2 CT findings of 332 adult malrotation patients

\begin{tabular}{ll}
\hline & $\begin{array}{l}\text { Total }(\boldsymbol{n}=\mathbf{3 3 2}) \\
\text { No. (\%) }\end{array}$ \\
\hline CT classification & \\
Duodenal partial rotation & $225(67.8)$ \\
$D_{Y} J_{R} C_{R}$ & $12(3.6)$ \\
$D_{Y} J_{R} C_{L}$ & $4(1.2)$ \\
$D_{Y} J_{R} C_{M}$ & $2(0.6)$ \\
$D_{Y} J_{R} C_{P}$ & \\
Duodenal nonrotation & $63(19.0)$ \\
$D_{N} J_{R} C_{R}$ & $15(4.5)$ \\
$D_{N} J_{R} C_{L}$ & $5(1.5)$ \\
$D_{N} J_{R} C_{P}$ & \\
Duodenal rotation & $1(0.3)$ \\
$D_{Y} J_{M} C_{R}$ & $3(0.9)$ \\
$D_{Y} J_{L} C_{R}$ & $2(0.6)$ \\
$D_{Y} J_{L} C_{L}$ & \\
Position of SMA relative to SMV & $165(49.7)$ \\
Left rear & $73(22.0)$ \\
Left & $66(19.9)$ \\
Left front & $28(8.4)$ \\
Right rear & $11(3.3)$ \\
Whirlpool sign &
\end{tabular}

$S M A$ superior mesenteric artery, SMV superior mesenteric vein

There was no significant difference in gender distribution or mean age between the two groups $(p=0.76$ and 0.89 , respectively). Abdominal pain was a common symptom in both groups $(p=0.45)$. The incidence of intestinal obstruction and volvulus was significantly higher in the surgically managed group compared to the conservatively managed group ( $p=0.002$ and 0.046 , respectively). Of the seven patients who developed volvulus, none had SMA/SMV inversion, but all demonstrated SMV angulation. Besides, the "whirlpool sign" was observed in six of them, and volvulus was confirmed intraoperatively in the remaining one patient. There was no significant difference in the distribution of symptom durations between the two groups $(p=0.36)$, but half of the patients in the surgical group had chronic onset, while $24.1 \%$ of the patients in the conservative group showed acute symptoms (Additional file 1: Fig. S2). The CT classification of the two groups was also compared as shown in Fig. 5, which showed that the conservative group was predominantly $\mathrm{D}_{Y} \mathrm{~J}_{R} \mathrm{C}_{R}(64.5 \%, 51 / 79)$ (type $\left.4 \mathrm{a}-\mathrm{b}\right)$, while the surgical group was predominantly $\mathrm{D}_{\mathrm{N}} \mathrm{J}_{\mathrm{R}} \mathrm{C}_{\mathrm{R}}(41.7 \%, 5 / 12)$ (type $1 \mathrm{a}-\mathrm{b})$. There was a statistically significant difference in the CT classification of the two groups $(p=0.016)$ with the conservatively managed group demonstrating more heterogeneity. In addition, no abnormalities in the SMA/SMV relationship were observed in the surgically 


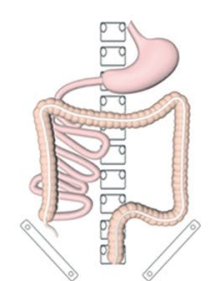

(1a)

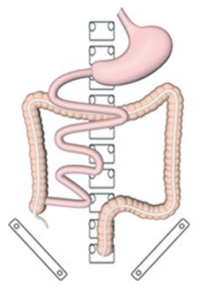

(4a)

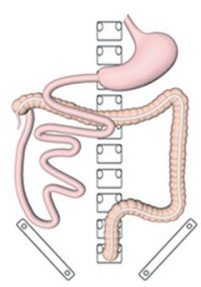

(4b)

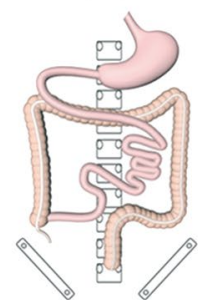

(8)

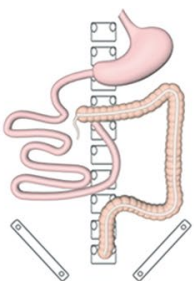

(1b)

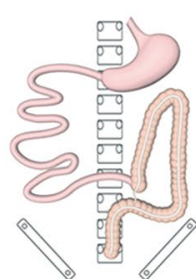

(2)

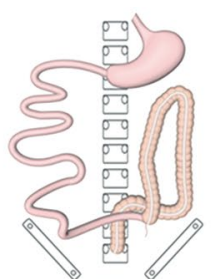

(3)

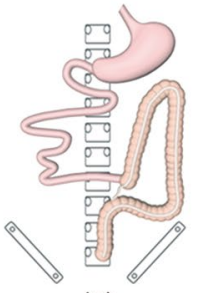

(5)

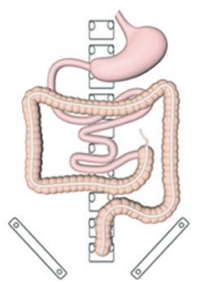

(9)

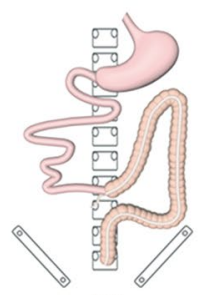

(6)

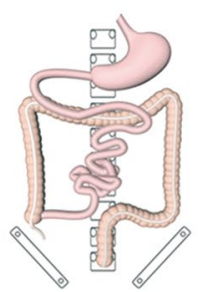

(10)

Fig. 2 Schematic diagram of the ten subtypes. (1)a) and (1) b) two cases of $D_{N} J_{R} C_{R}$ subtype: normal and high cecum; (2) $D_{N} J_{R} C_{L} ;(\mathbf{3}) D_{N} J_{R} C_{p} ;(\mathbf{4 a})$ and (4b) two cases of $D_{Y} J_{R} C_{R}$ subtype: normal and high cecum; (5) $D_{Y} J_{R} C_{L} ;(\mathbf{6}) D_{Y} J_{R} C_{M} ;(7) D_{Y} J_{R} C_{p} ;(8) D_{Y} J_{L} C_{R} ;(\mathbf{9}) D_{Y} J_{L} C_{L} ;(10) D_{Y} J_{M} C_{R}$

managed group. No statistical differences between the two groups $(p=0.75)$ were observed with respect to mesenteric vessel orientation.

\section{Discussion}

In this study, intestinal variations of malrotation were categorized into ten groups based on three common anatomical landmarks (duodenum, jejunum, and cecum) on CT images (Fig. 2), a classification scheme easily adaptable to clinical practice and easily understandable by clinicians. Furthermore, CT results from these patients were further analyzed with respect to their clinical manifestations and treatments received in an effort to facilitate identification of high-risk patients by their CT presentation, thus enabling individualized treatment and followups. To our knowledge, this is the largest clinical and radiological study of adult intestinal malrotation to date.

Compared with infants and children, the existence of adult intestinal malrotation has been historically ignored due to the lack of characteristic clinical features and is often described as an unexpected discovery in the literature $[19,20]$. However, the current study confirms that malrotation is not only a condition to be recognized by pediatricians, but also need to be taken into account by gastroenterologists and surgeons when diagnosing adult patients. In 332 adult patients, this abnormality occurred in any age (mean age $51.0 \pm 15.3$ years, range $18-97)$ and was twice as common in men (male/female: 1.91:1) a finding significantly different from previous studies (Durkin et al. 0.43:1 [5], Nehra et al. 0.78:1 [6], and Anand et al. 1:1 [4]).

Imaging studies of intestinal malrotation are infrequent, and studies with large sample sizes are even rarer. Long et al. [13] performed a typology of intestinal malrotation by analyzing the intestinal distribution on pediatric UGI series, after which Fay et al. [21] utilized radiationfree MRI for further study of malrotation in children, confirming its diagnostic reliability. However, the value of CT has traditionally been underestimated. Although the current gold standard for the diagnosis remains UGI series, a number of authors including Zissin et al. [19] have highlighted the unique advantages of abdominal CT in the diagnosis of malrotation in adults. In Yang et al. [18], CT manifestations associated with an increased incidence of complications were described; however, that work only included 14 patients. Based on the analysis of 332 patients in the present study, the advantages of $C T$ in detection of asymptomatic malrotation are further confirmed. Moreover, the $D_{N} J_{R} C_{R}$ subtype $(1 a-b)$ was most common in patients undergoing surgery, whereas the 

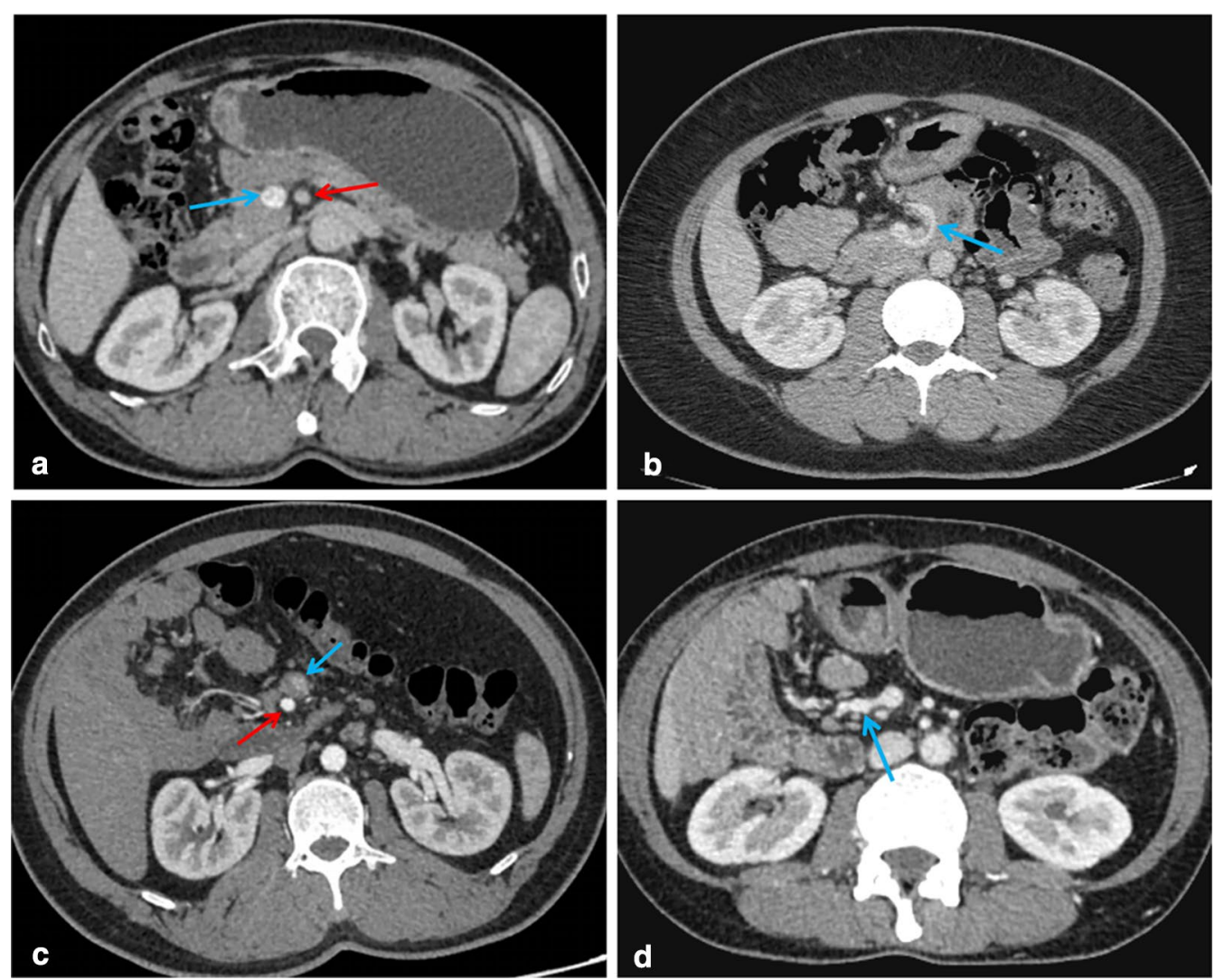

Fig. 3 Relationship between the superior mesenteric artery (red arrow) and superior mesenteric vein (blue arrow). a Normal (SMA on the left side of SMV); $\mathbf{b}$ the SMV forms an angle around the SMA toward the right abdominal cavity; c abnormal (inversion); $3 \mathrm{~d}$ : the SMV goes straight to the right abdomen

$\mathrm{D}_{\mathrm{Y}} \mathrm{J}_{\mathrm{R}} \mathrm{C}_{\mathrm{R}}$ subtype (4a-b) was most common in those managed conservatively. These findings may help clinicians detect more insidious forms of malrotation, guiding subsequent treatment and follow-up.

The current findings suggest that the presence of the jejunum in the right abdomen is a consistent feature of intestinal malrotation, present in $98.2 \%$ (326/332) of patients. Cecal ectopia was found in $12 \%$ (40/332) of patients with 29 cases located on the left, 7 in the pelvis, and 4 in the middle of the abdominal cavity, a more common finding than in Yang et al. (1/14) [18]. Cecal ectopia has been described in several case reports [22-25], and patients may develop atypical abdominal pain in the presence of concurrent appendicitis, requiring prompt identification. Sonomura et al. emphasized the high diagnostic value of coronal $\mathrm{CT}$ in acute appendicitis with intestinal malrotation [25] which seems reasonable considering the uncertainty regarding the location of the appendix in patients with malrotation. Although an abnormal SMA/ SMV relationship was only observed in $8.4 \%$ (28/332) of patients, 20 cases were asymptomatic (Additional file 1: Table S1). Abnormal SMV angulation was observed in
98.7\% of patients with an otherwise normal SMA/SMV relationship. Anatomic inversion between SMA and SMV found on CT and US has been proposed as a feature of intestinal malrotation [26], but this abnormal anatomical relationship has been shown to be inadequate in the diagnosis of isolated malrotation (observed in only $28 \%$ of patients with malrotation) [16] and can also be observed in normal people [27]. Therefore, in the diagnosis of malrotation, not only the relative position of the SMA and SMV should be noted, but also abnormal angulation of the SMV.

Furthermore, 91 patients with detailed clinical information and treatment history were analyzed. Not surprisingly the incidence of both intestinal obstruction and volvulus were significantly higher in the surgically managed group ( $p=0.002$ and 0.046 , respectively). In addition, although the mean age of the two groups was similar, the conservatively managed group encompassed a wider age range. Older patients in particular were more likely to be treated conservatively, consistent with previous findings [28]. This may be attributable to older patients, in general, being poorer surgical candidates. 


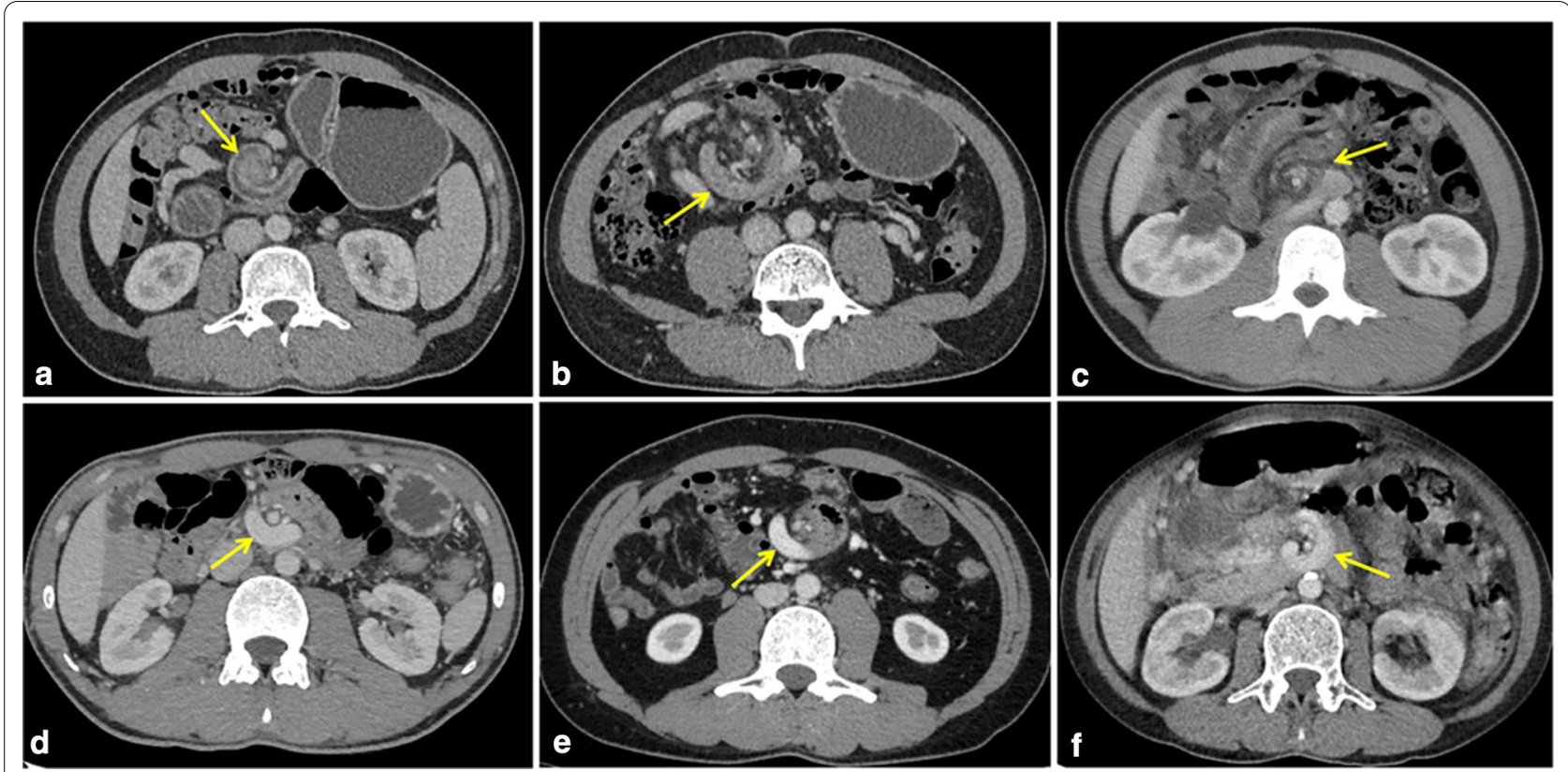

Fig. 4 "Whirlpool sign" in different patients. a, b A 34-year-old male with volvulus from the surgical group ( $D_{Y} J_{R} C_{R}$, type $\left.4 a\right)$. The images show torsion of duodenum, proximal jejunum and mesenteric vessels (arrow). c $A$ 30-year-old male with volvulus from the surgical group ( $D_{Y} J_{R} C_{M}$, type 6). $\mathbf{d}$, e Two males with volvulus from the conservative group $\left(D_{Y} J_{R} C_{R}\right.$, type $\left.4 a\right)$. The degree of torsion of vessels and intestines is mild (arrow). $\mathbf{f} A$ 51-year-old asymptomatic female $\left(D_{Y} J_{R} C_{R}\right.$, type $\left.4 a\right)$. The portal vein rotates anterior to the superior mesenteric artery

Interestingly, although half of the patients in the surgical group had a short duration of illness, 33.3\% (4/12) also had a duration of illness longer than one month and two of them even longer than one year. In addition patients in the conservative treatment group had more symptoms compared to the surgical group. This could be due to two factors: The conservative group had a larger number of cases, and patients in it generally had a longer duration of disease, which makes them more likely to have various gastrointestinal symptoms. Although further analysis showed that this difference was not statistically significant, such factors should still be taken into account.

Significant differences in CT classification were observed between the two groups. $D_{Y} J_{R} C_{R}$ subtype (4a-b) was present in up to $64.5 \%$ of the conservatively managed group but only in $25 \%$ of the surgically managed group $(p=0.013)$. In Yang et al., volvulus occurred more frequently in patients with partial rotation of the duodenum and colon [18], consistent with the current results. However, although six of the seven patients $\left[D_{Y} J_{R} C_{R}\right.$ (Fig. 2, type 4a-b): $5, D_{Y} J_{R} C_{M}$ (Fig. 2, type 6): 1 , and $D_{N} J_{R} C_{R}$ (Fig. 2, type 1a): 1] who developed volvulus exhibited duodenal partial rotation, four of them with $D_{Y} J_{R} C_{R}$ subtype had less severe volvulus that improved with conservative treatment. These results suggest that patients with a partially rotated duodenum are less likely to require surgery when the cecum is in a normal position. But the small number of patients with volvulus in both studies limits the strength of these findings. Moreover, although none of the patients with volvulus had an abnormality in the relative position of SMV and SMA, we observed SMV angulation in all of their CT images, and in addition, most of them (6/7) showed the typical "whirlpool sign." Previous studies have confirmed the sensitivity of the "whirlpool sign" in CT and US images in the diagnosis of volvulus [12, 29], and the current study also supports this.

Another unexpected finding in the current study was that the proportion of asymptomatic patients was as high as $56.6 \%(188 / 332)$, much higher than the $17 \%(14 / 82$, $p<0.001)$ in a previous study [6]. Schey et al. [30] have suggested that patients with duodenal nonrotation are more often asymptomatic, which is further confirmed by the radiological study by Zissin et al. [19]. However, in the current study, only $19.1 \%(36 / 188)$ of asymptomatic patients exhibited duodenal nonrotation, and further, such patients tended to have a variety of other intestinal abnormalities (Additional file 1: Table S1). In addition, the "whirlpool sign" was observed in four asymptomatic patients, which mainly showed mild rotation of the vessels and mesentery, without intestinal torsion, explaining their lack of symptoms upon presentation. In fact, many asymptomatic patients diagnosed incidentally can recall bouts of abdominal discomfort potentially related to 
Table 3 Clinical and CT characteristics of surgical and conservative groups

\begin{tabular}{|c|c|c|c|}
\hline Parameters & $\begin{array}{l}\text { Surgical } \\
\text { group } \\
(n=12)\end{array}$ & $\begin{array}{l}\text { Conservative } \\
\text { group }(n=79)\end{array}$ & $p$ \\
\hline Gender ratio (male/female) & $2: 1$ & $1.47: 1$ & .76 \\
\hline \multicolumn{4}{|l|}{ Age, years } \\
\hline Mean & $50.4 \pm 17.5$ & $51.2 \pm 18.0$ & .89 \\
\hline Range & $26-73$ & $18-84$ & \\
\hline \multicolumn{4}{|l|}{ Symptoms } \\
\hline Abdominal pain & $11(91.7)$ & $63(79.7)$ & .45 \\
\hline Bloating & $7(58.3)$ & $19(24.1)$ & \\
\hline Vomiting & $3(25.0)$ & $14(17.7)$ & \\
\hline Nausea & $3(25.0)$ & $3(3.8)$ & \\
\hline Diarrhea & - & $5(6.3)$ & \\
\hline Constipation & - & $2(2.5)$ & \\
\hline Intestinal obstruction $(n=19)$ & $7(58.3)$ & $12(15.2)$ & .002 \\
\hline Complication: volvulus $(n=7)$ & $3(25.0)$ & $4(5.1)$ & .046 \\
\hline Duration of symptoms & & & .36 \\
\hline Hours/days & $6(50.0)$ & $19(24.1)$ & \\
\hline Weeks & $2(16.7)$ & $14(17.7)$ & \\
\hline Months & $2(16.7)$ & $22(27.8)$ & \\
\hline Years & $2(16.7)$ & $24(30.4)$ & \\
\hline CT classification ${ }^{\ddagger}$ & & & .016 \\
\hline Duodenal partial rotation & & & .33 \\
\hline$D_{Y} J_{R} C_{R}$ & $3(25.0)$ & $51(64.5)$ & .013 \\
\hline$D_{Y} J_{R} C_{L}$ & $1(8.3)$ & $1(1.3)$ & \\
\hline$D_{Y} J_{R} C_{M}$ & $1(8.3)$ & $1(1.3)$ & \\
\hline$D_{Y} J_{R} C_{P}$ & $1(8.3)$ & - & \\
\hline Duodenal nonrotation & & & .52 \\
\hline$D_{N} J_{R} C_{R}$ & $5(41.7)$ & $20(25.3)$ & \\
\hline$D_{N} J_{R} C_{L}$ & - & $2(2.5)$ & \\
\hline$D_{N} J_{R} C_{P}$ & - & $1(1.3)$ & \\
\hline Duodenal rotation & & & .44 \\
\hline$D_{Y} J_{L} C_{R}$ & $1(8.3)$ & $1(1.3)$ & \\
\hline$D_{Y} J_{L} C_{L}$ & - & $2(2.5)$ & \\
\hline Position of SMA relative to SMV & & & .75 \\
\hline Left rear & 7 & 34 & \\
\hline Left & 3 & 27 & \\
\hline Left front & 2 & 12 & \\
\hline Right rear & 0 & 6 & \\
\hline Whirlpool sign & 2 & 4 & \\
\hline
\end{tabular}

SMA superior mesenteric artery, SMV superior mesenteric vein

${ }^{\ddagger}$ Fisher's exact test was used to determine significance of the difference in CT classification between the two groups $(p=.016<0.05)$

their malrotation if their past symptoms are thoroughly interrogated $[2,31]$. In the present study, the majority of asymptomatic cases, except for some diagnosed incidentally during routine physical examinations, were detected as a result of an examination or treatment for coexisting disease, and even if there were symptoms that might be associated with malrotation, it was difficult to definitely attribute them as such. In another CT study [19], the proportion of asymptomatic patients also reached $94.4 \%$ (17/18). However, these patients likewise could have been only temporarily asymptomatic or only had only mild symptoms that were insufficiently severe to report. Yet, these individuals would still potentially be at risk for future volvulus. The incidence of volvulus in the current study was only $2.1 \%(7 / 332)$, much lower than the $12 \%$ in Nehra et al. $(n=82, p<0.001)$ [6]. The increasing adoption of abdominal CT may improve reader confidence in identifying asymptomatic cases of malrotation, thus reducing the proportion found in the setting of volvulus.

Currently, the Ladd's procedure is a widely accepted standard treatment for intestinal malrotation, and some studies have emphasized that the Ladd's procedure should be routinely used in patients with intestinal malrotation, regardless of age [32, 33]. However, in the present study, the rate of surgery was only $13.2 \%(12 / 91)$, much lower than previously reported (Additional file 1 : Fig. S3), and of the 12 patients who underwent surgery, only three were treated with Ladd's procedure (Fig. 6), which seems to suggest an overall inadequate understanding of the disease and its treatment. In fact, although surgery is sometimes necessary, it also should be pursued cautiously. Compared to infants and children, adult patients are more likely to have major postoperative complications and have a higher reoperation rate $[5$, 34]. In addition, some patients with intestinal necrosis will undergo partial small bowel resection. In this setting, inadequate identification or evaluation of malrotation preoperatively may lead to a more complicated surgery and adverse outcomes. Hsu et al. have reported on short bowel syndrome caused by undiagnosed intestinal malrotation resulting from inappropriate surgical maneuvers and emphasize that CT should be utilized to adequately evaluate each patient preoperatively, especially for procedures with limited field of view, such as laparoscopic and Da Vinci robotic surgery [35]. Given the increasing popularity of minimally invasive surgery, number of asymptomatic patients with malrotation, and various malrotation subtypes, adequate scrutiny of the proximal bowel and mesenteric vessels on preoperative $\mathrm{CT}$ is increasingly important.

The current study has several limitations. First of all, although 332 patients were included in this study, detailed clinical data could only be examined in 91 . However, this remains the largest study of adult malrotation to date. Secondly, a large number of patients were asymptomatic; although, since this was a retrospective study spanning 7 years and most patients had comorbidities, this was difficult to truly validate. In addition, we must emphasize that the $\mathrm{CT}$ classification 

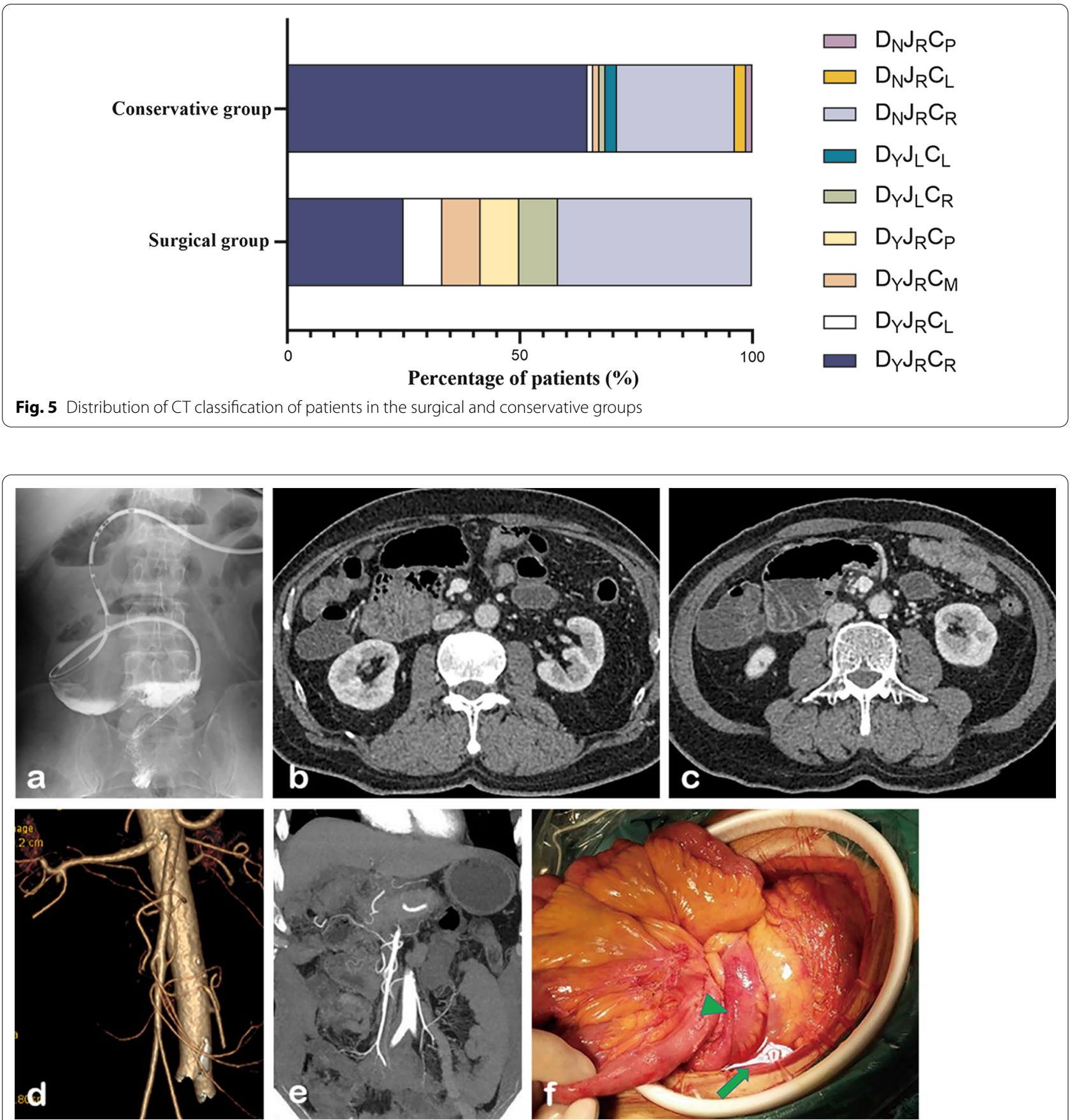

Fig. 6 A 64-year-old man developed volvulus after radical resection of rectal cancer $\left(D_{N} J_{R} C_{R}\right.$, type 1a). a Endoscopic catheterization: After passing through the duodenum, which was located to the right of the midline, the catheter was unable to pass smoothly into the misplaced obstructed proximal jejunum. b, c CT showed that the duodenum and jejunum were located in the right upper abdomen. $\mathbf{d}$, e CTA shows SMA and its branches. $6 f$ : Ladd's bands were found during surgery (arrow). The terminal ileum was visualized (arrowhead)

we proposed is a summary description of common anatomical features and the anatomy of the same subtype may also vary, which requires a specific analysis by the radiologist. Finally, follow-up data could not be reliably obtained, preventing assessment of the effects of various treatments. Long-term follow-up of such patients will be crucial in future studies.

In summary, adult intestinal malrotation is much more common than typically considered. CT not only allows the detection of a greater number of asymptomatic 
patients but also facilitates diagnostic precision enabling classification of malrotation into subtypes.

\author{
Abbreviations \\ SMA: Superior mesenteric artery; SMV: Superior mesenteric vein; UGI: Upper \\ gastrointestinal.
}

\section{Supplementary Information}

The online version contains supplementary material available at https://doi. org/10.1186/s13244-021-00999-3.

Additional file 1. Supplementary Methods: Diagnostic criteria for intestinal malrotation. Table S1: CT findings of 188 asymptomatic patients. Fig. S1: Flowchart for inclusion of patients. Fig. S2: Duration of symptoms in the 91 patients for whom detailed clinical data were available. Fig. S3: Comparing the treatment modality of patients in our study to previous studies.

\section{Acknowledgements}

We thank Xinyueyuan Hao for sorting the details of data.

\section{Authors' contributions}

Yaqi Shen was involved in conceptualization, methodology, and funding acquisition; Ziman Xiong was involved in methodology, formal analysis, data curation, writing —original draft, and visualization; John N. Morelli was involved in validation and writing — review and editing; Zhen Li was involved in writing - review and editing and funding acquisition; Xuemei Hu was involved in investigation and funding acquisition; and Daoyu Hu was involved in supervision and funding acquisition. All authors read and approved the final manuscript.

\section{Funding}

This work was supported by the grants from National Natural Science Foundation of China (NSFC) Nos. 82071890, 82071889, 81801695, 81771801, and 81571642 .

\section{Availability of data and materials}

The datasets used or analyzed during the current study are available from the corresponding author on reasonable request.

\section{Declarations}

\section{Ethics approval and consent to participate}

This retrospective study was approved by the Ethics Committee of Tongji Hospital, and the informed consent of all patients was waived.

\section{Consent for publication}

This manuscript is approved by all patients for publication.

\section{Competing interests}

All authors declare no conflict of interest in the present study.

\section{Author details}

${ }^{1}$ Department of Radiology, Tongji Hospital, Tongji Medical College, Huazhong University of Science and Technology, 1095 Jiefang Avenue, Qiaokou District, Wuhan 430030, Hubei, China. ${ }^{2}$ Department of Radiology, St. John's Medical Center, Tulsa, OK, USA.

Received: 11 January 2021 Accepted: 21 April 2021

Published online: 30 April 2021

\section{References}

1. Aboagye J, Goldstein SD, Salazar JH et al (2014) Age at presentation of common pediatric surgical conditions: reexamining dogma. J Pediatr Surg 49(6):995-999

2. Powell DM, Othersen HB, Smith CD (1989) Malrotation of the intestines in children: the effect of age on presentation and therapy. J Pediatr Surg 24(8):777-780

3. Applegate KE (2009) Evidence-based diagnosis of malrotation and volvulus. Pediatr Radiol 39(Suppl 2):S161-S163

4. Anand U, Kumar R, Priyadarshi RN, Kumar B, Kumar S, Singh VP (2018) Comparative study of intestinal malrotation in infant, children, and adult in a tertiary care center in India. Indian J Gastroenterol 37(6):545-549

5. Durkin ET, Lund DP, Shaaban AF, Schurr MJ, Weber SM (2008) Age-related differences in diagnosis and morbidity of intestinal malrotation. J Am Coll Surgeons 206(4):658-663

6. Nehra D, Goldstein AM (2011) Intestinal malrotation: varied clinical presentation from infancy through adulthood. Surgery 149(3):386-393

7. Alessandri G, Amodio A, Landoni L, Carino NDL, Bassi C (2016) Recurrent acute pancreatitis in bowel malrotation. Eur Rev Med PharmacoL 20(22):4719-4724

8. Kotobi H, Tan V, Lefèvre J, Duramé F, Audry G, Parc Y (2017) Total midgut volvulus in adults with intestinal malrotation. Report of eleven patients. J Visc Surg. 154(3):175-183

9. Graziano K, Islam S, Dasgupta R et al (2015) Asymptomatic malrotation: Diagnosis and surgical management: an American Pediatric Surgical Association outcomes and evidence based practice committee systematic review. J Pediatr Surg 50(10):1783-1790

10. Draus JMJ, Foley DS, Bond SJ (2007) Laparoscopic Ladd procedure: a minimally invasive approach to malrotation without midgut volvulus. Am Surg 73(7):693-696

11. Birajdar S, Rao SC, Bettenay F (2017) Role of upper gastrointestinal contrast studies for suspected malrotation in neonatal population. J Paediatr Child H 53(7):644-649

12. Shimanuki Y, Aihara T, Takano H et al (1996) Clockwise whirlpool sign at color Doppler US: an objective and definite sign of midgut volvulus. Radiology 199(1):261-264

13. Long FR, Kramer SS, Markowitz RI, Taylor GE (1996) Radiographic patterns of intestinal malrotation in children. Radiographics 16(3):547-56; discussion 556-60

14. Sizemore AW, Rabbani KZ, Ladd A, Applegate KE (2008) Diagnostic performance of the upper gastrointestinal series in the evaluation of children with clinically suspected malrotation. Pediatr Radiol 38(5):518-528

15. Dilley AV, Pereira J, Shi EC et al (2000) The radiologist says malrotation: Does the surgeon operate? Pediatr Surg Int 16(1-2):45-49

16. Esposito F, Vitale V, Noviello D et al (2014) Ultrasonographic diagnosis of midgut volvulus with malrotation in children. J Pediatr Gastr Nutr 59(6):786-788

17. Zheng Z, Huang J, Wei H, Liu J, Huang Y (2014) Adult congenital intestinal malrotation accompanied by midgut volvulus: report of eight cases. Int J Clin Exp Med 7(6):1614-1618

18. Yang B, Chen W, Zhang X, Luo Z (2013) Adult midgut malrotation: multidetector computed tomography (MDCT) findings of 14 cases. Jpn J Radiol 31(5):328-335

19. Zissin R, Rathaus V, Oscadchy A, Kots E, Gayer G, Shapiro-Feinberg M (1999) Intestinal malrotation as an incidental finding on CT in adults. Abdom Imaging 24(6):550

20. Pickhardt PJ, Bhalla S (2002) Intestinal malrotation in adolescents and adults: spectrum of clinical and imaging features. AJR Am J Roentgenol 179(6):1429-1435

21. Fay JS, Chernyak V, Taragin BH (2017) Identifying intestinal malrotation on magnetic resonance examinations ordered for unrelated indications. Pediatr Radiol 47(11):1477-1482

22. Kharel H, Pokhrel NB, Kharel Z, Sah D (2020) A case report on left-sided appendicitis with intestinal malrotation. Cureus 12(1):e6687

23. Shilling Bailey K, Rokosz J (2019) Acute appendicitis in a patient with situs inversus totalis, intestinal malrotation, and congenitally corrected transposition of the great arteries. Am Surg 85(8):e398-e399

24. Chuang PW, Huang B, Liu CH, Chen C, Tsai M (2015) Left-sided appendicitis in an elderly patient with midgut malrotation. Indian J Surg 773:S1418-S1420 
25. Sonomura T, Koyama T, Ishii S et al (2014) Acute appendicitis with intestinal malrotation: the usefulness of coronal computed tomography. Internal Med 53(14):1511-1513

26. Nichols DM, Li DK (1983) Superior mesenteric vein rotation: a CT sign of midgut malrotation. AJR Am J Roentgenol 141(4):707-708

27. Zerin JM, DiPietro MA (1991) Mesenteric vascular anatomy at CT: normal and abnormal appearances. Radiology 179(3):739-742

28. Husberg B, Salehi K, Peters T et al (2016) Congenital intestinal malrotation in adolescent and adult patients: a 12-year clinical and radiological survey. SpringerPlus 5(1):66

29. Yeh WC, Wang HP, Chen C, Wang HH, Wu MS, Lin JT (1999) Preoperative sonographic diagnosis of midgut malrotation with volvulus in adults: the "whirlpool" sign. J Clin Ultrasound 27(5):279-283

30. Schey WL, Donaldson JS, Sty JR (1993) Malrotation of bowel: variable patterns with different surgical considerations. J Pediatr Surg 28(1):96-101

31. Firor HV, Steiger E (1983) Morbidity of rotational abnormalities of the gut beyond infancy. Cleve Clin Q 50(3):303-309
32. Cohen Z, Kleiner O, Finaly R et al (2003) How much of a misnomer is "asymptomatic" intestinal malrotation? Isr Med Assoc J 5(3):172-174

33. Maxson RT, Franklin PA, Wagner CW (2003) Malrotation in the older child: surgical management, treatment, and outcome. Am Surg 61(2):135-138

34. Ferreira MS, Simões J, Folgado A et al (2020) Recurrent midgut volvulus in an adult patient-The case for pexy? A case report and review of the literature. Int J Surg Case Rep 66:91-95

35. Hsu Y, Hsu L, Huang W, Weng J, Chen C (2018) Short bowel syndrome caused by laparoscopic loop enterostomy of the jejunum in an adult with undiagnosed intestinal malrotation. Medicine 97(40):e12712

\section{Publisher's Note}

Springer Nature remains neutral with regard to jurisdictional claims in published maps and institutional affiliations.

\section{Submit your manuscript to a SpringerOpen ${ }^{\circ}$ journal and benefit from:}

- Convenient online submission

- Rigorous peer review

- Open access: articles freely available online

- High visibility within the field

- Retaining the copyright to your article

Submit your next manuscript at $\boldsymbol{\nabla}$ springeropen.com 